Jens S. Dangschat

\title{
Sag' mir, wo Du wohnst, und ich sag' Dir, wer Du bist!
}

Zum aktuellen Stand der deutschen Segregationsforschung

\section{Einleitung}

Das Zusammenleben von Menschen war immer mit einer Hierarchisierung des Raumes verbunden. Personen, die ein hohes Ansehen genießen, wurde immer bereitwillig der beste Platz eingeräumt, von dem man alles hörte und sah, wo man selbst gesehen wurde, von wo aus das Wort zu ergreifen leichter war. Aber nicht nur die Welt des Mikro-Raumes war und ist zu Zeiten des Höhlenlebens und moderner Verwaltungen in Hierarchien organisiert, auch der Meso- und der Makro-Raum wird diesen anerkannten sozialen Abstufungen unterzogen. Die »feinen Leute« wohnten immer im Vorderhaus, während sich Arbeiter und Gesinde mit den dunklen Terrassen, Hinterhöfen und Gängen bescheiden mußten. Reiche wohnten immer dort, wo die Luft am saubersten, die Ruhe am gesichersten, die Nachbarn am auserwähltesten waren. Bourdieu $(1991,30)$ drückt dies als das Bemühen um unterschiedliche Formen von Lokalisationsprofiten aus:

- um Situationsrenditen (Nähe zu erwünschten Personen und Dingen und Ferne zu unerwünschten Personen und Dingen; Zuwachs an Ruhe, Ästhetik und Sicherheit),

- um Okkupations- oder Raumbelegungsprofite (Quantität des verbrauchten (Wohn)raums, unverbaubares Blickfeld) sowie

- um Positions- oder Rangprofite (Adresse) als besonderer Form des symbolischen Kapitals.

»Der soziale Raum ${ }^{1}$ weist eine Tendenz auf, sich mehr oder weniger strikt im physischen Raum in Form einer bestimmten distributionellen Anordnung von Akteuern und Eigenschaf-

1 Mit »sozialem Raum « umschreibt Bourdieu (1982) das System sozialer Hierarchien, das sich aus der Menge und Zusammensetzung von "ökonomischem Kapitak (Geld und Vermögen) und »kulturellem Kapital« (formale Bildung, Besitz von Kulturgütern und kulturelle Kompetenzen) ergibt. In diesem System sind Klassen- und Klassenfraktionen vor allem über die Zugehörigkeit zu Berufen verortet. »Raum« ist hier also nicht physikalisch $\mathrm{zu}$ verstehen, sondern entspricht einer ideellen Vorstellungswelt, einer Modellierung sozialer Ungleichheit innerhalb einer Gesellschaft. 
ten niederzuschlagen. ... Daraus folgt, daß der von einem Akteur eingenommene Ort und sein Platz im angeeigneten physischen Raum hervorragende Indikatoren für seine Stellung im sozialen Raum abgeben« (Bourdieu 1991, 25).

Damit ist das Konzept der »residentiellen Segregation« angesprochen, also der ungleichen Verteilung von Wohnorten unterschiedlicher sozialer Kategorien (Klassen, Schichten, Haushalts- oder Milieutypen, Altersgruppen sowie ethnische und rassische Gruppen) im Raum. Es ist eines der zentralen und der ursprünglichen Forschungsthemen aller »Schulen« der Stadtund Regionalsoziologie. Im krassen Widerspruch zur hohen Bedeutung, die eine ungleiche Partizipationsschance am verstädterten Raum und damit zur sozialen und sozialräumlichen Ausdifferenzierung moderner Gesellschaften hat, steht jedoch der relativ geringe Ertrag dieser Forschungen.

Ziel sozialwissenschaftlicher Bemühungen sollte es sein, ein gesellschaftliches Phänomen in ausreichender Differenzierung beschreiben, sein $\mathrm{Zu}$ standekommen begründen, seine Folgen abschätzen und künftige Entwicklungen vorhersagen zu können. Je nach wissenschaftlicher Position gehört dazu, die bestehende Situation derart zu beeinflussen, daß negativ eingeschätzte Entwicklungen beendet und verhindert, positive Entwicklungen dagegen unterstützt werden.

Von einer Segregationstheorie wird also gefordert, daß mit ihr das Phänomen der ungleichen Verteilung gesellschaftlicher Gruppen im Raum angemessen beschrieben und erklärt werden kann, daß die Folgen der Segregation benannt und künftige Entwicklungen antizipiert werden können und schließlich sollte das Phänomen auch bewertet werden, um gegebenenfalls Vorschläge zur Beeinflussung zu unterbreiten.

Um den aktuellen Stand der deutschen Segregationsforschung (der sich nicht wesentlich vom internationalen Standard unterscheidet) darlegen und kritisch einordnen zu können, werden zu den genannten Aspekten die Erkenntnisse - nach Schulen geordnet - dargestellt und bewertet (Kap. 3-5). Danach werden - als Reaktion auf die formulierte Kritik - zentrale Forschungsfragen benannt (Kap. 6). Zuvor soll jedoch noch einmal zurückgeblickt und gefragt werden, was die ursprüngliche Intention des Segregationskonzeptes war (Kap. 2).

\section{Der Ursprung des Konzepts der residentiellen Segregation}

Karl Marx, Émile Durkheim, Max Weber und Georg Simmel waren die ersten Soziologen, die Überlegungen zur sozialen Ungleichheit aus dem Sozialen Wandel ableiteten und in Typologien faßten: Klassengegensätze, unterschiedliche Positionen in einem zunehmend arbeitsteilig organisierten Produktionsproze $\beta$ mit entsprechend differenzierten Belohnungen, der Hinweis auch auf die kulturelle Dimension neben der ökonomischen, den Lebensstil und die Lebensführung. 
Ausgehend von diesen Überlegungen zur sozialen Ungleichheit entwickelte der amerikanische Soziologe Robert Ezra Park Mitte der 20er Jahre das Konzept der residentiellen Segregation (vgl. Park 1924, 1925). Dazu übertrug er die Annahmen Durkheims, Webers und Simmels zur sozialen Ungleichheit in den Raum, indem er die physische Distanz zwischen Wohnstandorten $^{2}$ als Indikator sozialer Distanz ansah. ${ }^{3}$ Er nutzte die Methodologie Durkheims der Überführung nicht beobachtbarer sozialer Tatbestände (soziales Konstrukt) auf beobachtbare soziale Phänomene (Indikator). Weiter übernahm er das Phänomen der wsozialen Schließung« von Max Weber und übertrug es idealtypisch in den Raum, indem er unterstellte, daß die soziale Segregation (Distinktion) zu städtischen Teilgebieten führe, die nach der Sozial- und Baustruktur nahezu homogen wären. Diese Gebiete nannte Park - unter explizitem Rückgriff auf Webers Idealtypus - 'natural area'. Eine perfekte residentielle Segregation einer städtischen Gesellschaft und der städtischen Räume ist demnach dann gegeben, wenn eine Stadt aus nach der Sozial-, Bau- und funktionalen Struktur homogenen, aber untereinander verschiedenen Teilgebieten besteht. Mit dem Idealtypus ist jedoch verbunden, daß erstens die behaupteten Sachverhalte empirisch eher nicht in dieser Weise auftreten und zweitens, daß der theoretische Fortschritt aus der Interpretation des Abweichens von diesem Idealtypus erzielt wird. Die Forschungsfrage lautet also: Was bewirkt, daß der Idealzustand perfekter Segregation als 'natural areas' nicht eintritt?

Damit war das Konzept der residentiellen Segregation als einem (gut zu messenden) Indikator sozialer Segmentation entwickelt. Es soll nicht mehr und nicht weniger leisten, als (städtische) soziale Ungleichheit durch ihr Abbild im (städtischen) Raum zu analysieren. Diese Ansicht der nahezu unmittelbaren Übertragung sozialer Ungleichheit in den Raum findet auch heute noch Zuspruch in der Sozialökologie (Herlyn 1974b, c, Dangschat 1990, Friedrichs 1977, 1995), in der polit-ökonomischen Geographie (Dear/ Wolch 1991, Krätke 1995) und in der Kultursoziologie (Bourdieu 1991). Eine Segregatiostheorie besteht demnach aus drei Elementen (vgl. Dangschat 1985, 31-38):

- Einer Theorie zur Beschreibung und Erklärung sozialer Ungleichheit (zum aktuellen Stand vgl. Hradil 1987, 1992, Berger/Hradil 1990, Müller 1992, Berger/Vester 1997),

2 Deshalb wird hier auf dem Begriff »residentielle Segregation « bestanden und gegen den Begriff »soziale Segregation« abgegrenzt. Letzterer ist deshalb unscharf, weil er sich mit der Segmentation, der sozialen Abgrenzung sozialer Gruppen (beispielsweise durch Vermeidung sozialer Kontakte) überschneidet. Ist abweichend nur von »Segregation« die Rede, so ist diese Begrifflichkeit immer im Sinne residentieller Segregation zu verstehen.

3 Friedrichs (1995: 80) ergänzt diese Sichtweise auf der Makro-Ebene, indem er die Möglichkeit anspricht, »... das Ausmaß der Segregation auch als Indikator der gesellschaftlichen Integration einer sozialen Gruppe« zu interpretieren. 
- einer Theorie zur Beschreibung und Erklärung räumlicher Ungleichheit (zum aktuellen Stand vgl. Hamm 1982, Läpple 1991, Dangschat 1994a, 1996), - einer Theorie zur Beschreibung und Erklärung der Zuweisungsprozesse sozialer Gruppen in spezifische Räume, in der Weise, daß sich die soziale Ungleichheit weiter verschärft (vgl. Marcuse 1989, Krätke 1995).

\section{Sozialökologischer Ansatz}

\section{I Beschreibung der Segregation}

Die Erkenntnisse der amerikanischen Stadt- und Regionalsoziologie der 20er bis 60er Jahre, und damit auch der Segregationsforschung, wurden in der Bundesrepublik durch Vertreter der Sozialökologie übernommen (vor allem durch Friedrichs, Hamm und Herlyn). Ausgehend von Übersetzungen amerikanischer Originalliteratur (Atteslander/Hamm 1974a, Herlyn 1974a) und einer Darstellung des US-amerikanischen Forschungsstandes (Friedrichs 1972, als Vorläufer zu Friedrichs 1977) wurde die sozialökologische Stadtsoziologie der Chicagoer Schule und ihrer amerikanischen Nachfolger als (Groß-)Stadtsoziologie eingeführt, welche die bis dahin domierende Gemeindesoziologie ablöste. Hamm (1977) und Friedrichs (1977) gaben dann fast zeitgleich die ersten eigenständigen Texte bundesdeutscher Sozialökologie heraus.

Die amerikanische Segregationsforschung hatte sich jedoch mittlerweile deutlich von den Ursprüngen Parks entfernt. Aufgrund der Entwicklung flächendeckender, regelmäßig erhobener Statistiken von Stadtregionen und der Entwicklung der elektronischen Datenverarbeitung sowie unterstützt von der seit den 40er Jahren in den USA dominierenden Systemtheorie und den Theorien »mittlerer Reichweite«, hatte sich der Schwerpunkt der Segregationsforschung auf die Entwicklung von Segregationsindizes konzentriert (vgl. zur Definition und Diskussion der wichtigsten Indizes Blasius 1988). Diese Indizes geben das Maß der ungleichen Verteilung sozialer Gruppen in einer Stadt an. Mittels des Vergleichs der Indexwerte über die Zeit und/oder zwischen Städten wurde versucht, empirische Regelmäßigkeiten zu entdecken, d.h. Theorien zu bilden.

Das empirische Ergebnis einer Fülle von Fallstudien war, daß die sozialen Gruppen am Rande der sozialen Hierarchien ${ }^{4}$ die höchsten Indexwerte aufweisen, ihre Distinktion also am stärksten durch räumliche Absonderung ${ }^{5}$

4 Dabei wird soziale Ungleichheit bis heute entweder in Kategorien der sozialen Schichtung (SES und seine Einzelindikatoren: Bildung, Berufsposition, Einkommen), des Lebenszyklus (Alter, Haushaltstyp), der Nationalität resp. des »Gastarbeiter«-Status und von Lebensstilen analysiert.

5 Die Kategorie »Raum « wird theoretisch nicht berücksichtigt; der Raum geht - ganz in der Tradition des Container-Konzepts - als Träger spezifischer Merkmale ein und dient le- 
demonstrieren, während mittlere Lagen am wenigsten segregiert sind ${ }^{6}$. Daraus ergibt sich eine u-förmige Verteilung der Segregationsindex-Werte über die in eine Rangordnung gebrachten sozialen Gruppen. Eine weitere Erkenntnis besteht darin, daß die Indexwerte über die Zeit größer werden, was als Folge einer die Modernisierung begleitenden Arbeirsteilung interpretiert wurde.

Der Nachteil aller Indizes - neben der Abhängigkeit von der Größe der räumlichen Sub-Einheiten - ist, daß ihnen nicht anzusehen ist, aufgrund welcher Konzentrationen der Durchschnittswert zustande kommt. ${ }^{7}$ Eine Segregationsforschung, die das sozialräumliche Phänomen der Segregation auf einen Indexwert und dessen Analyse als eine pauschale Aussage über eine gesamtstädtische Situation reduziert, steckt daher zwangsläufig in einer Sackgasse theoretischer Entwicklung.

Eine innerhalb der Sozialökologie bedeutsame Theorie ist die Sozialraumanalyse (vgl. Shevky/Bell 1955, Hamm 1977). Ihre zentrale These ist, »daß die städtischen Phänomene ... auf regionaler Basis die Veränderungen in der Gesamtgesellschaft widerspiegelten« (Shevky/ Bell 1974, 125). Die Sozialraumanalyse ist also eine Theorie des Sozialen Wandels, von dem einerseits angenommen wird, daß er sich in der sozialräumlichen Strukturierung der Städte niederschlägt, andererseits ist aber die Stadtentwicklung nur dann verstehbar, wenn sie als Teil eines übergeordneten Sozialen Wandels begriffen wird:

"Großstadt (ist) ein Produkt der komplexen Gesamtheit der modernen Gesellschaft: Folglich können die sozialen Formen des städtischen Lebens nur im Zusammenhang mit dem sich wandelnden Charakter der größeren umfassenden Gesellschaft verstanden werden « (Shevky / Bell 1974, 126).

"Aus der Analyse der sozialen Trends in Städten ergeben sich drei Faktoren - soziale Position, Verstädterung und Segregation ${ }^{8}$ - die unserer Meinung nach grundlegend für die städtische Differenzierung und soziale Schichtung sind« (Shevky/Bell 1974, 130).

Diese Faktoren sind ausreichend und notwendig, um die meisten der festgestellten Variationen zwischen den Bevölkerungsgruppen bestimmter Gebiete zu erklären. Gleichzeitig führen diese Faktoren zu spezifischen und

diglich als zweidimensionales Muster positiver und negativer Konstellationen der Sozial-, Infra- und Baustruktur.

6 Ein Teil dieser Erkenntnis ist jedoch ein statistisches Artefakt, weil die beiden Extremgruppen nur über je eine Nachbargruppe verfügen.

7 Z.B. kann die relativ geringfügige Abweichung aller Sub-Einheiten vom städtischen Durchschnitt zum gleichen Indexwert führen wie die starke Abweichung nur weniger Teilräume. Beide Fälle stehen jedoch für eine deutlich andere reale Form der Segregation.

8 Unter »Segregation« wird in der Sozialraumanalyse sowohl bei Shevky/Bell, als auch bei Friedrichs die sozialräumliche Trennung ausschließlich von ethnischen Gruppen verstanden. 
konstanten Verteilungsmustern. Während sich die "soziale Position« in Segment-Strukturen räumlich ausdifferenziert (analog zu dem Stadtentwicklungsmodell von Homer Hoyt), folgt die »Verstädterung « einer konzentrischen Differenzierung (anaiog zum Modell von Emest W. Burgess). Die Verteilung der Ethnien folgt hingegen einem eher dispersen Muster, was eine Parallele zum Mehrkernmodell von Harris und Ullman darstellt.

Die weltweit stabile Faktor- und Raumstruktur wurde wiederholt als Beweis der Güte dieser "Theorie «" angesehen (vgl. Abu-Lughod 1969) ${ }^{10}$. Aufgrund der häufig verletzten impliziten Annahmen in den einzelnen Fallbeispielen muß jedoch geschlossen werden, daß die mathematische Methode offensichtlich stabiler ist als die Theorie oder die Realität.

Im Zuge der Perzeption der amerikanischen Sozialökologie in Deutschland wurden die empirischen Ergebnisse der Analyse US-amerikanischer Städte bruchlos übertragen und mit den gleichen Annahmen und Methoden in Deutschland überprüft. Die Ursache hierfür war die theoretische Grundhaltung des kritischen Rationalismus, der von der Annahme ausgeht, daß sich Regressionen und Korrelationen als soziale Gesetze formulieren lassen, die eine raum- und zeitunabhängige Gültigkeit aufweisen. Empirisch vorfindbare Unterschiede seien vor allem auf unterschiedliche Phasen einer prinzipiell nach gleichem Muster verlaufenden Entwicklung und auf Besonderheiten des konkreten sozialräumlichen Kontexts zurückzuführen (vgl. z.B. Dangschat et al. 1985). Während der erste Faktor praktisch der Kem stadtsoziologischer Theorien sei, ist der zweite durch sog. Anschlußtheorien und durch die Berücksichtigung lokaler Spezifika zu analysieren.

Die Leistung der bundesdeutschen Sozialökologie bestand in einer expliziten Formalisierung und Operationalisierung der zu beobachtenden Sachverhalte (vgl. Friedrichs 1977, 216-281). Dies führte zu einer Reihe empirischer Beschreibungen, ${ }^{11}$ erbrachte aber keine eigentlichen Fortschritte zur Erklärung der Phänomene. Durch die Übernahme amerikanischer Methoden und Techniken sowie der impliziten Annahmen der reinen Marktwirtschaft im Wohnungssektor und der amerikanischen Struktur sozialer Ungleichheit auf bundesdeutsche Verhältnisse wurde im Gegenteil sehr lange ein theoretischer Fortschritt verhindert.

9 Diese Theorie ist jedoch fragwürdig, wenn sie zu jedem Zeitpunkt für jedes politische, ökonomische, soziale und kulturelle Umfeld zu ähnlichen Ergebnissen führt.

10 Friedrichs (1977) hat beispielsweise auf Basis der Volkszählung von 1970 für die Region Hamburg eine vergleichbare Faktorenanalyse durchgeführt und kommt zu fünf Faktoren: Urbanismus, Sozialer Rang, Mobilität, bauliche Dichte und Segregation, die in der Region Hamburg $69 \%$ und in der Stadt Hamburg $83 \%$ der Varianz erklären.

11 Vgl. kritisch dazu Atteslander/Hamm (1974b, 15): »Eine Summienung empirischen Materials über Gemeinden allein führt noch keineswegs zu einer Theorie der Gemeinde.« 
Auch die »Gentrification « als spezielle Form der Segregation ${ }^{12}$ wurde als Invasion der sog. Pioniere, Gentrifier und UItra-Gentrifier (vgl. Dangschat 1988, Dangschat/Friedrichs 1988, Blasius/Dangschat 1990, Alisch 1993, Blasius 1993, Alisch/Dangschat 1996) betrachtet. Dabei wurden diese Personengruppen über das gleichzeitige Auftreten bestimmter Merkmale definiert (Syndrom-Ansatz) und der Zusammenhang zu bestimmten Einstellungen, Lebensweisen und Lebensstilen überprüft. Hier fanden zudem erste Öffnungen zu Überlegungen der Raumstruktur sowie der Beeinflussung des Prozesses durch Marktmechanismen, Makler- und Investoren-Interessen sowie der ambivalenten Regulation durch den »lokalen Staat《 statt (vgl. Dangschat 1991, Alisch/Dangschat 1996).

\subsection{Ursachen der Segregation}

Auf der Basis vor allem US-amerikanischer Städte wurden »Theorien« der residentiellen Segregation entwickelt, die sich inhaltlich jedoch auf die Kausalstrukturen zwischen flächendeckenden Merkmalen der Volkszählungen und der statistisch erfaßbaren Daten der Städte (als Kontexte) beschränken. Damit wird die Theoriearbeit auf Dimensionen reduziert, die sich aus der Tradition von Statistikern ergeben. Aspekte der sozialen Ungleichheit werden in der Regel auf demographische Daten verkürzt, die Theoretisierung der Bedeutung des Raumes wird auf städtische Teilgebiete als Zähleinheiten und Träger von Merkmalen reduziert. Zuweisungsprozesse zwischen räumlichen Einheiten und sozialen Gruppen finden keine Berücksichtigung. Diese Modelle sind daher sinnleer und geben kaum Hinweise über die Ursachen der Segregation oder die Prozesse, die die sozialräumliche Ungleicheit bestimmen (kritisch dazu: Blasius/Dangschat 1991).

Ein weiterer Aspekt ist die Analyse von 'tipping points' (vgl. Schelling 1971), also der Frage, ab welcher Konzentration einer als niedrig eingestuften sozialen Gruppe (meist: Ethnien oder Rassen) es dazu kommt, daß der Auszug von Alteingesessenen eine Dynamik erhält, die zu unumkehrbaren Sukzessionen führt. Diese Untersuchungen wurden auch in Deutschland durchgeführt (z.B. Hoffmeyer-Zlotnik 1977, Kecskes/Knäble 1988) und ergaben Anteile - meist von Türken -, die bei Überschreitung zu einem deutlich intensiveren Auszug von Deutschen aus dem Wohnhaus oder dem Quartier führen. Das Ergebnis der Distinktion sozialer Gruppen ist immer

12 »Gentrification« ist einerseits die ökonomische Aufwertung des innerstädtischen Wohnungsbestandes durch sog. Luxusmodernisierung und Umwandlungen der Miet- in Eigentumswohnungen sowie den Zuzug höherer sozialer Schichten, oft verbunden mit der Sukzession der Alteingesessenen und andererseits die sozio-kulturelle Umwertung durch »neue« Haushaltsformen, nicht-bürgerliche Lebensstile und Inhaber moderner Dienstleistungsberufe. 
eine Segregation, die höher liegt, als es die Bewohner aufgrund ihrer Präferenzen und Toleranzniveaus selbst für erforderlich halten

Esser (1988) und Coleman (1990) folgend, hat Friedrichs zuletzt seine Vorstellungen einer Segregationstheorie als eine Mikro-Makro-Theorie modelliert (Friedrichs 1988c, 1995, 90-98). Dabei werden die in der Sozialökologie traditionellen Zusammenhänge zwischen Merkmalen auf der »Makro《-Ebene (je höher die Einkommensungleichheit, desto umfangreicher die Segregation) ${ }^{13}$ aufgelöst, indem die Zusammenhänge auf der Mikro-Ebene (je größer die individuellen Wahlmöglichkeiten, desto eher können Wohnstandortwahlen nach eigenen Präferenzen vorgenommen werden) empirisch überprüft und durch Kontakthypothesen (je höher die Einkommensungleichheit, desto größer die individuellen Wahlmöglichkeiten) resp. Aggregationsregeln (je umfangreicher Standortwahlen nach eigenen Präferenzen vorgenommen werden können, desto umfangreicher die Segregation) mit der Makro-Ebene verbunden werden.

So richtig es ist, die Position zurückzuweisen, man könne die Gerichtetheit von Korrelationen als Theorie interpretieren (was häufig "Standard « innerhalb der Sozialökologie ist), so hat die von Friedrichs vorgeschlagene Theorie dennoch mindestens drei Defizite:

(1) Der Raum wird weder in seiner ungleichen Struktur, noch gar als Hebel gesellschaftlicher Regulation oder in seiner Funktion zur Reproduktion sozialer Ungleichheit wahrgenommen; er hat paradoxerweise in einer Theorie, die die sozialräumliche Ungleichheit zum Gegenstand hat, keine explizite Bedeutung.

(2) Die soziale Ungleichheit wird auf die Einkommensungleichheit reduziert (die ihrerseits nicht erklärt oder auch nur eingeordnet wird). Auch wenn die zentrale Bedeutung dieses Merkmales für soziale Ungleichheit in einer kapitalistischen Gesellschaft nicht bestritten wird, übersieht ein solcher Ansatz die aktuelle Diskussion sozialer Ungleichheit, die - bei allen Unterschieden zwischen der These der Entstrukturierung und dem Wiede-

13 Friedrichs (1995: 90) kritisiert, daß es sich bei makrosoziologischen Studien der Segregation oft um fragwürdige Korrelationen auf Stadtteilebene aggregierter Individualmerkmale handele. Diese werden häufig als Indikatoren für individuelles Handein oder von Präferenzen interpretiert (was das Risiko des ökologischen Fehlschlusses beinhaltet). Im Widerspruch zu dieser nachvollziehbaren Kritik steht jedoch seine Einschätzung der Modelle von Marshall/Jiobu (1975) und Roof et al. (1976) als »sehr gut«. M.E. sind solche Theorien eher unergiebig, die - wie Marshall/Jiobu - ausschließlich den Anteil an Nichtweißen, die Zahl der Nichtweißen oder das Differential des Bevölkerungswachstums beider Gruppen als angebliche Ursachen von Segregation heranziehen. Angeregt durch diese wenig sinnvollen Modelle hatte Friedrichs (1977: 281) ein eigenes MakroModell entwickelt, in dem der Anteil an Gastarbeitern, die Größe einer Stadt (die eine berufliche Differenzierung nach sich zieht, die zu einer Einkommensdifferenzierung führt), die Reliefenergie und die Ausstattung der Teilgebiete (die beide zu ungleichen Mieten führen) als Ursachen von Segregation aufgeführt wird. 
rentdecken von Klassenstrukturen (vgl. Dangschat 1997a) - sich in der Tatsache einig ist, daß die Reduktion sozialer Ungleichheit auf das Einkommen unzureichend ist. Selbst Friedrichs $(1995,92)$ geht von indirekten Effekten aus, indem er behauptet, daß das Ausmaß sozialer Ungleichheit die Differenzierung der Lebensstile nach sich zieht, die wiederum den Wunsch nach räumlicher Nähe zu Personen ähnlichen Lebensstils vergrößert ${ }^{14}$.

(3) Die zur Erklärung herangezogenen Merkmale auf der Makro-Ebene sind völlig unzureichend. Sie umfassen nicht das Entstehen sozialer Ungleichheiten (die, wie erwähnt, über die Einkommensungleichheiten hinausgehen) oder räumlicher Hierarchisierung aus strukturellen und institutionellen Zusammenhängen resp. der Dynamisierung des Sozialen Wandels. Weitere eigenständige deutsche Beiträge in der Segregationsanalyse sind die Anwendung des heuristischen, multivariaten Verfahrens der Korrespondenzanalyse, um die gleichzeitige Wirkung mehrerer Faktoren auf Segregationsmuster und Segregierungen zu analysieren (vgl. Dangschat/Blasius 1987, Blasius/Dangschat 1991). Diese Methode ist - im Gegensatz zur französischen Soziologie - in Deutschland noch immer gering verbreitet. Auf diese Weise wurde der Zusammenhang zwischen Merkmalen der Sozialstruktur, der Wohnbaustruktur und von geographischer Nähe analysiert.

Einen zweiten Schritt stellt schließlich ein Segregations-Index (index of inequality, II) dar, der es - in Anlehnung an den Dissimilaritätsindex von Duncan - erlaubt, die Segregation in nur einer Stadt ${ }^{15}$ in ein Kausalmodell zu integrieren (vgl. Blasius/Dangschat 1991). Damit wird es möglich, das Ausmaß der Segregation einer sozialen Gruppe (gegenüber einer anderen oder dem Rest der Wohnbevölkerung) auf einzelne Faktoren zurückzuführen. Auch wenn dieses ein deutlicher Fortschritt innerhalb positivistischer Erklärungen ist, bleiben die grundsätzlichen Probleme bestehen, wie die Abhängigkeit von der Größe der räumlichen Einheiten und von dem durch die amtliche Statistik bereitgestellten, eingeschränkten Set an flächendeckenden Statistiken.

Einen Erklärungsansatz residentieller Segregation aus »Faktoren der Wirtschafts- und Sozialstruktur« deutet Herlyn $(1974 b, 24)$ zumindest als ein »Syndrom aus verschiedenen Faktoren« an:

14 Damit faßt Friedrichs Lebensstil als Indikator sozialer Ungleichheit auf, der gleichsam soziale Ungleichheit sichtbar macht und damit zur Orientierung an »den Gleichen« in Alltagspraktiken herangezogen werden kann. Dieser enge empirische Zusammenhang wird jedoch verschiedentlich massiv bestritten (Hradil 1987, Hitzler 1994).

15 Für die bisherigen Indizes konnte eine Varianz nur durch Einbeziehung mehrerer Städte erzeugt werden. Eine »Erklärung《 der residentiellen Segregation für mehrere Städte ist jedoch theoretisch sinnlos, weil unter vergleichbaren Rahmenbedingungen (Makro-Kontexte) gerade die varianzerzeugende Ungleichheit zwischen den Städten (aufgrund der lokalstaatlichen Regulation und der Spezifik der Meso- und Mikroebene) Gegegenstand einer Segregationstheorie sein sollte, die aber auf der Suche nach den Gemeinsamkeiten aller Städte verlorengeht. 
»Knappheit des Bodens im Zusammenhang mit individualistischem Bodenrecht und Bodenspekulation, sich dabei ergebende Verdrängungskonkurrenz, standardisierte Bauprogramme, Kriegsfolgen, das in technischen Kategorien verhaftete Denken der Planer, die klassen- bzw. schichtspezifische Statussuche bei Tendenzen zur sozialen Mobilität und der zirkuläre Prozeß von Diskriminierung und Stigmatisierung.« (Herlyn 1974c, 95)

Im Rahmen der Siedlungssoziologie wird die Vergesellschaftung und Vergemeinschaftung im Raum durch eine Theorie der sozialen und räumlichen Organisation erklärt (vgl. Atteslander/Hamm 1974b, Hamm 1982, 22-37). Die Siedlungssoziologie ist gegenüber der Gemeinde- und Sozialökologie die umfassendere Theorie,

" ... weil sie die verschiedensten Arten der Raumaneignung, der Raumnutzung sowie der Raumgestaltung in Wechselwirkung mit dem Sozialverhalten von Individuen und Gruppen und der Funktion von Institutionen zu erklären hat. Nicht die einzelne Siedlung, sondern die Besiedlung eines bestimmten Raumes insgesamt muß Objekt der Siedlungssoziologie sein. Nicht eine Siedlungseinheit ist Erkenntnisobjekt wie in der Gemeindesoziologie, sondern der Raumbezug der Gesellschaftu (Atteslander/Hamm 1974b, 16).

Dem französischen Ansatz der Morphologie (Durkheim, Halbwachs), der Anthropogeographie Ratzels und den ursprünglichen Ideen Parks folgend, konzipieren Atteslander und Hamm ein System der Mensch-Umwelt-Beziehungen, das aus vier Subsystemen besteht (dem physisch-räumlichen, dem psycho-physischen, dem technologischen und dem sozio-kulturellen Subsystem), die sich wechselseitig in Richtung eines Gleichgewichts der Mensch-Umwelt-Beziehungen regulieren.

Für sozialwissenschaftliche Fragestellungen ist das sozio-kulturelle Subsystem maßgeblich, das Atteslander und Hamm als ein Raum-VerhaltenSystem bezeichnen. Dieses Subsystem unterteilen sie wiederum in drei weitere Subsysteme: ein morphologisches, ein semiotisches und ein institutionelles Subsystem, wobei sich die drei Subsysteme über die Zeit wechselseitig verändern.

Das morphologische Subsystem wird durch (natural) areas gebildet, also durch Gebiete, die bezüglich der Nutzung und der Nutzer relativ homogen sind. Hier wird explizit der Bezug zu den ersten Arbeiten der Chicagoer Schule der Sozialökologie gewählt und gleichzeitig deren und ihrer Nachfolger empirizistische Verwirrungen kritisiert. Als Kriterium der Abgrenzung morphologischer Subsysteme werden unterschiedliche Interaktionsdichten herangezogen.

Das institutionelle Subsystem besteht aus sozialen Institutionen im Sinne der Theorie des Symbolischen Interaktionismus. Das können sein: »akzeptierte Verhaltensmuster, Werthierarchien, die Kosten-Nutzen-Relation, Bündel sozialer Normen, die soziale Kontrolle und die damit verbundenen Positionen, aber auch Macht und Legitimität und, damit verbunden, Entscheidungsstrukturen « (Atteslander/Hamm 1974b, 29). Damit sind verschiedene Lebensstile, Kalküle und Wertmuster, Tauschwert und Gebrauchswert, Recht- 
sprechung und -überwachung, (lokale) Politik und Raumplanung von der Konzeption her in dieses Subsystem integriert. Insgesamt sind in dem institutionellen Subsystem alle raumdeterminierten und raumdeterminierenden Handlungsweisen und Verhaltensformen auf der Mikro- (individuelle Handlungen), Meso- (kollektives Handeln von Gruppen und Personenaggregaten) und Makro-Ebene (institutionelles Handeln) zusammengefaßt.

Im semiotischen Subsystem werden schließlich die sozialpsychologischen Perzeptions- und Kognitionsprozesse verortet. Die Symbolik des Ortes, die sich vordergründig in der Ästhetik der Architektur resp. in der städtebaulichen Gestalt ausdrückt, ist jedoch gesellschaftlich ausgebildet. Sie unterscheidet sich in der Bedeutung, die ihr der Sender (Architekt, Planer, Bauherr) gab und der Interpretation der Empfänger, deren Dechiffrierung sozial differenziert ausfällt. Dem umwelt- oder sozialpsychologischen Ansatz von Barker folgend, gehen Atteslander und Hamm im Sinne einer Schließung von einer Synomorphie von Raumgestalt und Verhalten aus.

Atteslander und Hamm stellen damit die soziale Bedeutung des Raumes für die Segregation heraus. Konsequenterweise distanziert sich Hamm später von dem in Segregationstheorien überwiegenden »naiven« Raumverständnis eines Behälterraumes:

»Wenn nun Raum nichts anderes wäre als ein bloßes Gefäß, in dem soziale Beziehungen ablaufen, die von ihm gänzlich unabhängig sind, dann ist Raum allenfalls auf einer deskriptiven Ebene interessant, aber völlig unfruchtbar für die Erkenntnis dessen, worauf es einer solchen Soziologie ankäme: von sozialer Organisation nämlich« (Hamm 1982, 24) (Herv. i. Orig.). Und weiter: "So kann man sagen, daß es keinen Raum gibt, der nicht erst durch Wahrnehmung (und Bewertung, J.S.D.) soziale Bedeutung erlangte. Eben darin liegt die soziologische Bedeutung des Raumes begründet, daß er nicht existiert außer in unserer Wahrnehmung und daß diese Wahrnehmung immer und unausweichlich durch soziale Bezüge vorgeformt und vermittelt stattfindet. Damit wird Raum zu einer soziologischen Kategorie« (ebd., 26) (Herv. i. Orig.).

Hamm greift später nicht mehr auf das Mensch-Umwelt-System zurück, sondern verortet die physikalischen Aspekte des Raumes eindeutig im morphologischen Subsystem des Raum-Verhalten-Systems, wobei Morphologie als »Oberflächenbeschreibung des materiellen Substrats (Durkheim) von Räumen « (Hamm 1982, 27 Herv. i. Orig.) verstanden wird und » ... die drei Elemente materielles Substrat, Institutionen und Zeichen in einem nur analytisch trennbaren Abhängigkeitsverhältnis zueinander stehen « (ebd., 28). Damit stellt er den Raum als untrennbar von den sozialen Bezügen über alle Interaktionsformen dar.

\subsection{Folgen der Segregation und deren Bewertung}

Die Folgen der Segregation werden innerhalb der Sozialökologie überwiegend als negativ eingeordnet. Orientiert an Gans (1961) wird die Möglichkeit zur kulturellen und normativen Integration sozialer Gruppen als positiv 
und - der Vorstellung von Parks race-relation-cycle ${ }^{16}$ folgend - wird die räumliche Konzentration von Migranten als vorübergehend sinnvoll, notwendig und erwünscht angesehen. Gleichwohl überwiegt die negative Einschätzung, weil bei einer Segregation die Integration von Minoritäten in die Majoritätsgesellschaft erschwert oder verhindert werde. Das gilt nicht nur bezüglich der Migranten, sondern eben auch bezüglich der Orientierung unterer sozialer Schichten an bürgerlichen Verhaltensnormen und Wertestrukturen. Diese Einschätzung wird von der sog. »Kontakthypothese « geprägt, die jedoch in den unteren sozialen Rängen eher zu konfliktbeladenen Ausgrenzungen als zur Integration führt (vgl. Amir 1969, 338-340; Dangschat 1997b).

Gleichzeitig wirkt sich der systemtheoretische Hintergrund dahingehend aus, daß Gleichgewichtszustände angestrebt werden. Quantitativ wird die Segregation daher als Prozentsatz derer interpretiert, die umziehen müssen, um eine Gleichverteilung der sozialen Gruppen im Raum zu erzielen. Es ist jedoch zu fragen, warum eine Gleichverteilung als ideal angesehen wird, wo es doch zum Bestandteil großstädtischen Allgemeinwissens gehört, daß nicht alle mit allen zusammenleben wollen oder können. Für Herlyn (1974c, 102) »... erweist sich das Argument vom sozialen Aufstieg durch Mischung im Wohnbereich als ideologieverdächtig."

\subsection{Interventionen}

Da sich die Sozialökologie in der methodischen Ausrichtung dem Kritischen Rationalismus zuordnet, geht sie von einer »wertfreien« Position aus. Das bedeutet, daß sich Wissenschaft auf die Rolle einer exakten Beschreibung, des Testens von Hypothesen und des Modellierens statistischer Regelhaftigkeiten zu konzentrieren habe. Der Hinweis auf einen Prozentsatz einer sozialen Gruppe, der umzuziehen habe, weist jedoch auf implizite Wertungen hin, die beispielsweise von Kommunalpolitikern und Stadtplanern unmittelbar übernommen wurden. Insofern intervenieren Sozialökologen nach wie vor im Planungsdiskurs, auch wenn Friedrichs (1988b, 11) etwas resigniert resumiert:

»Die sozialwissenschaftliche Sehnsucht nach der Praxis, so berechtigt sie ist, dient tatsächlich eher der reformerischen Befriedigung und dem demonstrativen Engagement des Forschers einerseits und der Legitimation behördlichen Handelns andererseits - weniger den jeweils Betroffenen.«

Diese Position ist sicherlich nicht zu trennen von der enttäuschten Einsicht, daß die »... klassische Sozialökologie ... trotz der Erneuerungsversuche in

16 Park formulierte in den 30er Jahren die These des 'race-relation-cycle', d.h. zu Regelhaftigkeiten der schrittweisen Assimilation von Migranten. Die »Natürlichkeit« des räumlichen Ausschlusses von Migranten als wichtigen Schritt zur Integration/Assimilation ist jedoch bereits von Parks afro-amerikanischen Schülem bezweifelt worden 
den 40er und späten 50er Jahren als Theorie ausgedient « (Friedrichs, 1988b, 9) habe - was Herlyn (1990) vehement bestreitet. Friedrichs Skepsis gegenüber der Qualität einer Theorie, die er als wesentlicher Akteur selbst "importierte«, richtet sich gegen eine Stadttheorie, der der Gegenstand verlorengegangen ist (vgl. Saunders 1987).

In der Regel wurden als Ursache hierfür gesellschaftliche Ausdifferenzierungen angeführt, die die Erklärungskraft der »klassischen« Merkmale der Soziologie ausgehöhlt haben. Meines Erachtens nach ist es jedoch vor allem die Methode, die raum- und zeitunabhängige Beziehungen als Gesetzesaussagen (eines linear mathematisch-statistischen Zusammenhangs) zum Gegenstand hat, welche die Probleme erzeugt. Eine Stadtsoziologie, die auf diese Weise vom gesellschaftlichen und historischen Kontext abstrahiert, hat keine Möglichkeit die Spezifik einer eigenen Theorie zu entwickeln. Sie wird immer eine auf die Stadt als empirisch bestimmbarem Ort der intensivsten Moderne angewandte allgemeine Soziologie bleiben, ohne selbst in der Lage zu sein, die Spezifik des empirischen Ortes und des theoretischen Konstruktes Raum zu erfassen.

\section{Polit-ökonomischer Ansatz}

Der polit-ökonomische Ansatz wurde in den USA und in Europa in Opposition zu dem dominanten sozialökologischen entwickelt. Die Kritik richtete sich vor allem gegen dessen Schwerpunktsetzung auf der Deskription, gegen die mikro-soziologische Orientierung und die neopositivistische Methode (»Wissenschaft mit der Meßlatte «). Das betraf auch und gerade die Segregationsforschung, denn hier stehen sich zudem die fundamentalen Gegensätze eines Schichtungs- und eines Klassenansatzes gegenüber.

\subsection{Beschreibung der Segregation}

Aufgrund der makrotheoretischen Sichtweisen bleibt die Deskription der Segregierung im Rahmen dieses Ansatzes eher schwach. Vor allem der vereinfachende - und gegenüber der seinerzeitigen neomarxistisch/neoweberianischen Debatte um soziale Strukturierung deutlich veraltete - Ansatz sozialer Ungleichheit ging ursprünglich von zwei, später von drei Klassen aus, die sich gerade in solchen Städten, in denen das kapitalistische Verwertungsinteresse hoch ist, besonders deutlich verräumlichen und eine polarisierte Stadt - als 'dual city' (vgl. Blanke et al. 1986) resp. als 'dreigeteilte Stadt' (Häußermann/Siebel 1987, 1991a, Krätke 1991) - herausbilden.

In dieser Zwei-, später Dreiteilung wurden vor allem die Gründe für eine gesellschaftliche Spaltung erörtert (häufiger auch ohne den Wohnungsmarkt zu reflektieren), d.h. man ging davon aus, daß sich das Segregati- 
onsmuster bereits endgültig ausgebildet habe. Für die gesamte Stadtfläche interessierte man sich nicht - wie die Sozialökologie -, sondern nahezu ausschließlich für die Wohnstandorte der sozial Benachteiligten, der Marginalisierten, der Paupers des kapitalistisch-städtischen Modernisierungsprozesses. Es geht bei der Beschreibung daher weniger um Segregation, sondern um die räumliche Konzentration sozial Benachteiligter in benachteiligenden Wohn- und Wohnumfeldbedingungen (vgl. Alisch/Dangschat 1993).

Vor allem Häußermann und Siebel haben die sozialräumliche Ausdifferenzierung der Städte als dreigeteilt beschrieben. Dabei ist erstens nicht deutlich, ob damit die städtische Gesellschaft oder der städtische Raum gemeint ist, und zweitens, ob die Dreiteilung bereits eingesetzt hat resp. unter welchen Bedingungen sie sich entsprechend weiterentwickelt. Häußermann/ Siebel $(1987,138-148)$ trennen einerseits »Städte« voneinander ${ }^{17}$ :

"Die erste Struktur, die international wettbewerbsfähige Stadt, setzt sich aus den Glanz- und Höhepunkten einer Stadt zusammen, die überregionale Funktionen und Aufmerksamkeit auf sich ziehen können: Flughafen, internationaler Messe-, Kongreß- und Hotelbereich, aufwendige Freizeit- und Kultureinrichtungen, Verwaltungen international organisierter Unternehmen und Inseln luxuriösen Wohnens.«

Die »zweite« Stadt ist »... die normale Arbeits-, Versorgungs- und Wohnstadt für die deutsche Mittelschicht mit den Funktionen eines regionalen Oberzentrums..."

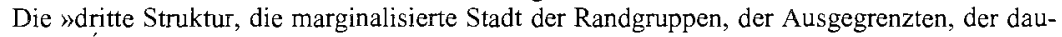
erhaft Árbeitslosen, der Ausländer, der Drogenabhängigen und der Armen.«

Sie räumen andererseits auch ein, daß »(v)erläßliche empirische Untersuchungen darüber, wie sich die soziale Segregation (gemeint ist: residentielle Segregation, J.S.D.) unter dem Einfluß der Arbeitsmarktkrise und Finanznot der Städte entwickelt hat, ... bisher nicht vor(liegen)« (Häußermann/Siebel 1987, 147). An anderer Stelle konzentrieren sie sich ausschließlich auf die gesellschaftlichen Ausdifferenzierungen. Auch hier gehen sie von einer Dreiteilung der (städtischen) Gesellschaft aus, wobei immer wieder von Schichtungen die Rede ist (vgl. Häußermann 1991, 94-95): ${ }^{18}$

》(E)in relativ kleines Segment höchst bezahlter Spitzenkräfte, deren Gehälter, Karrieremuster und Lebensstile sich an internationalen Märkten orientieren«,

»ein breites, aber eher rückläufiges Segment aus den Angehörigen der überwiegend deutschen Mittelschicht mit gesicherten Einkommen« und

17 In Häußermann/Siebel (1991b, 354-355) nehmen sie eine andere Dreiteilung der Städte vor, bei der die Kategorien der Distinktion ständig wechseln (ökonomische Nutzungen, Berufsgruppen, Nationalitäten, Bildung, Einkommen, Mieten, städtische Teilgebiete - es werden sogar ganze Städte benannt); dort wird noch deutlicher als im obigen Beispiel, daß es unklar ist, was genau mit dem Konzept der »dreigeteilten Stadt« gemeint ist. Auch bei deren Vorbild, der »quartered city《 von Marcuse (1989) gehen Merkmale der Städte und der Stadtbewohner unsystematisch in die Typologie ein.

18 Hierbei wird das Konzept der sozialen Ungleichheit nicht deutlich, außer, daß es einen Shift von der Mitte an die Ränder gibt, so daß es zu einer Dreiteilung kommt. Weitere Merkmale sozialer Ungleichheit werden von ihnen jedoch nicht berücksichtigt, obwohl sie weitere Distinktionskräfte berücksichtigen (s.u.). 
»ein schnell wachsendes Segment von am Rand der Gesellschaft lebenden, unqualifizierten Beschäftigten in der Grauzone des Arbeitsmarktes bzw. dauerhaft Arbeitslosen« (Häußermann/Siebel 1991a, 27).

Die Ausdifferenzierung der (städtischen) Gesellschaft wird vor allem durch drei Prozesse geprågt, die sich in

- Spaltungen zwischen Einheimischen und Zugewanderten,

- Spaltungen zwischen stabil im Arbeitsmarkt Verankerten und den Inhabern marginalisierter Jobs resp. den Arbeitslosen,

- kulturellen Ausdifferenzierungen (Heterogenisierung) zeigen, die weitgehend auf mittlere soziale Lagen und jüngere Kohorten begrenzt sind; sie äußern sich in einer Ausdifferenzierung von Lebensstilen, Haushalts- und Wohnformen.

Häußermann/Siebel $(1991 \mathrm{a}, 28)$ benennen schließlich in einem analytischen Zugang drei grundlegende Spaltungslinien moderner Stadtgesellschaften:

»Die ökonomische Spaltung nach Eigentum, Einkommen und Position auf dem Arbeitsmarkt; die soziale Spaltung nach Bildung, sozialer Integration und Position auf dem Wohnungsmarkt; die kulturelle Spaltung nach ethnischen Zugehörigkeit, Religion und normativen Orientierungen. ${ }^{19}$

Wie sich diese drei Dimensionen überlagern, wie sich die Sortierungsprozesse konkret im Raum darstellen und wie dieses wiederum als Reproduktion sozialer Ungleichheit fungiert, wurde - im Gegensatz zu den ähnlichen sozialökologischen Ansätzen - von Häußermann und Siebel jedoch nicht thematisiert, nicht zuletzt auch, weil die Bedeutung des Raumes von ihnen nicht reflektiert wird.

Läpple (1991) hat demgegenüber als Ergebnis einer umfangreichen Diskussion der historischen Entwicklung des Raumverständnisses in der Volkswirtschaftslehre, der Physik und der Geographie ein Konzept des »gesellschaftlichen Raumes« entwickelt, daß sich von dem bislang vorherrschenden »Container «-Konzept ${ }^{20}$ unterscheidet, indem es insbesondere die Produktions-, aber auch die Reproduktionsbedingungen des sozialen Konstrukts »Raum《 umfaßt. Ohne sich direkt auf das Raum-Verhalten-System von Hamm (1982) zu beziehen, kommt er jedoch zu ähnlichen Elementen eines Raumverständnisses für sozialwissenschaftliche Zwecke:

19 Interessant ist, daß Häußermann/Siebel - ohne jedoch darauf einzugehen - mit ihrem Vorgehen auf exakt die gleichen Dimensionen der Segregation kommen wie Shevky/Bell mit ihrer Sozialraumanalyse; zudem ist die hohe Übereinstimmung mit den drei Kapitalarten der Klassenstrukturen bei Bourdieu (1983) auffällig.

20 Damit ist die Vorstellung gemeint, daß demographische, soziale, ökonomische, baustrukturelle Daten etc. in der Regel als Statistiken Orten zugeordnet werden. Das gesellschaftliche Raumkonzept geht jedoch von einem konfigurationellen Verständnis des funktionalen Zusammenhangs von Menschen und Dingen aus. 
Das materiell-physische Substrat: Es wird gesellschaftlich 'produziert' und besteht aus der von Menschen umgeformten Natur und den materiellen Nutzungsstrukturen sowie den Menschen in ihrer körperlich-räumlichen Leiblichkeit.

Die gesellschaftlichen Interaktions- und Handlungsstrukturen bzw. die gesellschaftliche Praxis der mit der Produktion, Nutzung und Aneignung des Raumsubstrats befaßten Menschen.

Ein institutionalisiertes und normatives Regulationssystem, das als Vermittlungsglied zwischen dem materiellen Substrat und der gesellschaftlichen Praxis seiner Produktion, Aneignung und Nutzung fungiert. Dieses Regulationssystem kodifiziert und regelt im wesentlichen den Umgang mit den raumstrukturierenden Artefakten.

Ein mit dem materiellen Substrat verbundenes räumliches Zeichen-, Symbol-und Repräsentationssystem.

Läpple benennt jedoch lediglich die Dimensionen des gesellschaftlichen Raumes und versäumt es, sie miteinander in Beziehung zu setzen. Darüber hinaus stellt er keine Verbindungen zu Theorien der sozialen Ungleichheit her; beispielsweise läßt sich hier das Bourdieusche Struktur-HabitusPraxis-Konzept sowie das symbolische Kapital anführen (vgl. Bourdieu 1983; vgl. zur Kritik Dangschat 1994a). In ein Konzept gesellschaftlichen Raumes - insbesondere zur Interpretation von Segregation sollten strukturelle und Milieu-Eigenschaften der segregierten Wohnbevölkerung ebenso hinzugezogen werden wie die qualitative Ausstattung der Wohnungs- und Infrastruktur (vgl. ausführlicher Dangschat, 1994b, 1996).

\subsection{Ursachen der Segregation}

Die Ursachen für die soziale und sozialräumliche Ausdifferenzierung städtischer Gesellschaften und Räume wird überwiegend im kapitalistischen Verwertungsprozeß gegenwärtiger Modernisierung sowie deren (lokal-) politischer Regulation gesehen. 'Global cities' und andere Städte, $\gg . .$. die ihre Politik darauf konzentrieren, in der Städtekonkurrenz eine Position auf den vorderen Plätzen der 'internationalen Liga' zu behaupten oder zu erringen, fördern eine Tendenz zur 'dreigeteilten Stadt'« (Häußermann/Siebel 1991 a, 27) - »Politik des radikalen Wachstums«.

Häußermann (199l) und Häußermann/Siebel (1987, 1991a, b) messen den Veränderungen auf den Arbeitsmärkten eine entscheidende Bedeutung für die erneute Zunahme sozialer Ungleichheit in großstädtischen Gesellschaften zu. Gleichzeitig konstatieren sie, vielfältige Filter und Netze, $\gg .$. die das Durchschlagen sozialer in räumliche Strukturen in der Bundesrepublik verzögern und die Muster verzerren« (Häußermann/Siebel 199lb, 355356). Der lokalen Regulation, insbesondere des Wohnungsmarktes, komme 
daher eine entscheidende Bedeutung für das Ausmaß der Segregierung zu. Eine Möglichkeit zum Abbau der Arbeitslosigkeit sehen sie in einer Modernisierung des Dienstleistungssektors. ${ }^{21}$

Krätke $(1995,162)$ sieht

»(d)as sozialräumliche Gefüge der Stadt ... insbesondere von den ökonomisch-sozialen Strukturbedingungen der Stadtentwicklung und den gesellschaftlichen Beziehungen zwischen städtischen Teilräumen - z.B. Arbeitsbeziehungen, Kontrollbeziehungen, quartiersübergreifende Handlungsstrategien sozialer Akteure - geprägt. ... (D)ie Determinanten der Prozesse sozialräumlicher Restrukturierung sind aber aus stadtökonomischer Perspektive in erster Linie über den Mechanismus des städtischen Wohnungs- und Bodenmarktes zu erfassen. Dabei ist wiederum der Zusammenhang zwischen regionalökonomischem Strukturwandel und städtischem Bodenmarkt ebenso wie der Zusammenhang zwischen städtischer Arbeitsmarkt- und Wohnungsmarktentwicklung zu beachten«.

Die Segregations-Struktur wird demnach in den wettbewerbsorientierten Städten sowohl durch die Polarisierung des Arbeitsmarktes, als auch die Deregulierung lokaler Politik bestimmt; sie prägt sich besonders aus in »Inseln der Gentrifizierung « und in »sozialen Brennpunkten«.

Unter regulationstheoretischer Prämisse haben Esser/Hirsch (1987) den Ursachenkomplex gegenwärtiger städtischer sozialräumlicher Heterogenisierung, Fraktionierung und Polarisierung beschrieben. In diesem Zusammenhang wird der Wettbewerbsorientierung der lokalen Politik als Reaktion auf die Herausfordungungen der Globalisierung in Folge der krisenhaften ökonomischen Umstrukturierung die hauptsächliche Verantwortung für soziale und räumliche Spaltungen in den Großstädten zugeschrieben.

\subsection{Folgen und Bewertung der Segregation}

Häußermann/Siebel (1991a, 29) weisen zu Recht darauf hin, daß die Separierung ausschließlich der unteren und mittleren sozialen Lagen diskutiert wird - die der oberen Einkommenskategorien wird als »natürlich«, selbstverständlich und nicht als städtebaulich relevant hingenommen und daher nicht hinterfragt. Dabei geht durch deren Konzentration nach Gesetzen der Lokalisationsprofite eine bedeutsame "Integrationsmasse« verloren. D.h., während die Reicheren sich der Integrationsarbeit über räumliche Nähe zu Migranten und/oder Randgruppen weitgehend entziehen, lastet die Herausbildung multikultureller Gesellschaften vor allem auf der unteren Mittelschicht, auf Anhängern »alternativer« Wohnformen und Lebensentwürfen (vgl. Dangschat 1997b).

Bei der Bewertung der Segregation schließen sich Häußermann und Siebel weitgehend der Argumentation von Gans (1961) an, der sowohl positive

21 Ihre wiederholt formulierten Schlußfolgerungen aufgrund der Sekundäranalyse des Vergleichs der Dienstleistungsstrukturen in den USA, in Schweden und der Bundesrepublik von Esping-Anderson bezüglich der »Wunderwaffe Frauenerwerbstätigkeit» sind jedoch höchst fragwürdig. 
(geringerer Konformitätsdruck insbesondere im Konsumbereich, zunehmend auch bezüglich ethnisch geprägter Alltagspraktiken, Aufstiegsorientierung, geringeres Labelling etc.), wie negative Aspekte (geringer Kontakt zu anderen Lebensformen, Wertemustern und Lebensstilen, Schließungsprozesse nach innen fördern die Konflikte an den 'Grenzen') der Segregation benennt, ohne allerdings zu einer Entscheidung zu gelangen.

Häußermann/Siebel $(1991 \mathrm{a}, 26)$ vertreten die These, daß insbesondere für

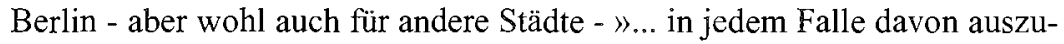
gehen (ist), daß die Zuwanderung nicht entsprechend der Aufnahmefähigkeit des Arbeitsmarktes dosiert werden kann.« Eine effiziente Kontrolle würde $» . .$. zu einem Überwachungsapparat führen, der sozial und politisch weder wünschbar noch akzeptabel wäre«. Bezüglich der Segregation nach ethnischen und rassischen Merkmalen nehmen sie Parks Position ein, wobei sie den Aspekt der Freiwilligkeit betonen. Segregationen von Migrantengruppen sollen weder durch »die Zwänge des Wohnungsmarktes« noch durch »soziale Diskriminierungen« diesen Gruppen aufgeherrscht werden (ebd., 32-33) - aber genau das ist der ausschließliche Grund für deren Segregation, wenn man die Aspekte des kulturellen Beharrens in 'ethnic neighbourhoods' als Reaktion auf Diskriminierungen und Assimilationen interpretiert.

Häußermann/Siebel (199la, 33) messen dem »... Abbau erzwungener und das Zulassen freiwilliger Segregation auf den Ebenen von Hausgemeinschaften, selbstgewählten Nachbarschaften und Wohnquartieren ...« eine entscheidende Bedeutung für eine positive Bewertung von Segregation zu. Auch wenn man diese Position ethisch teilt, scheint sie dennoch wenig operational und in konkreten Erhebungssituationen kaum bestimmbar zu sein (auch weil die Deutung der von Segregation Betroffenen dem Abbau eigener kognitiver Dissonanzen dient). Zudem ist zu berücksichtigen, daß erstens Orte sozialen Hierarchisierungen unterliegen und zweitens, daß ein »Konglomerat verschiedener Städte in einer « - auch wenn es »Inseln der Glückseeligen sind « - die soziale, kulturelle und funktionale Integration der Städte gefährden.

Vor dem Hintergrund allgemeiner Gentrifizierung innerstädtischer Wohngebiete kommen Alisch/Dangschat (1993) für die Armutsbevölkerung zu einer positiven Einschätzung von Segregationen unter der Bedingung, daß spezifische städtebaulich-funktionale Qualitäten und eine weitgend ausschließliche Einkommensarmut vorliegen (bei moderat hohem sozialen und kulturellen Kapital, vgl. Bourdieu 1983). Nur unter diesen Bedingungen und häufig unter Zuhilfenahme kommunikativer und partizipativer Techniken - kann das diskriminierte und verkümmerte soziale und kulturelle Kapital der Bevölkerung, deren ökonomisches Kapital kurz- und mittelfristig 
niedrig sein wird, so unterstützt werden ('empowerment'), daß neue Alltagspraktiken, mit Armut umzugehen ('coping'-Strategien), entwickelt werden können. Letzteres sind ausschließlich Interventionen in meist hoch segregierten Wohnquartieren (wie sie in lokalen Armuts»bekämpfungs«programmen resp. im Programm zur Intervention in 'Stadtteilen mit besonderem Erneuerungsbedarf' vorgenommen werden), ohne jedoch die Segregation selbst oder die Armut verringern zu können oder zu wollen.

\subsection{Intervention in die Segregierung}

Auch wenn der polit-ökonomische Ansatz im positivistischen Sinne nicht wertfrei ist und (unfreiwillige) Segregation abgelehnt wird, sind Hinweise in dieser Literatur selten, wie die Segregation zu verringern sei. Die oft strukturalistischen Ansätze beziehen sich demgegenüber auf die MakroEbene der Ursachen für eine soziale und/oder räumliche Ungleichheit. Im Rahmen einer allgemeinen Kapitalismuskritik wird die Art und Weise der Krisentegulation des ausgehenden Fordismus durch zunehmende Deregulierung des Arbeitsmarktes, der lokalen Politik, sowie die neo-liberalen Strömungen zunehmender 'private public-partnerships' und andere Formen der Flexibilisierung kritisiert. Die Kritik richtet sich gegen eine lokale Politik, die offener als je zuvor, mit einer »Politik der Lebensstile« die Welt der unternehmensbezogenen Dienstleister durch die Aufwertung der Innenstädte und ihrer angrenzenden attraktiven Wohngebiete fördert, hingegen Gebiete der 50er Jahre, weite Teile der weniger attraktiven innenstadtnahen Standorte und insbesondere die Großsiedlungen durch die Verweigerung von Reinvestitionen oder eine einseitige Belegungspolitik rasch herunterfiltern (vgl. Krätke 1991, Dangschat 1995).

Das Votum von Häußermann/Siebel (1991a) geht dahin, die Interventionen zum Abbau residentieller Segregation mittels einer »Verteilungspolitik « zu überdenken. Die insbesondere gegenüber Migranten angewandte Strategie diene tatsächlichen und/oder vermeintlichen Interessen der deutschen Bevölkerung und nicht der der Migranten. Eine Intervention bei freiwilliger Segregation solle ohnehin unterlassen werden. Aufgabe von Kommunalpolitik solle es vielmehr sein, die strukturellen Benachteiligungen der Marginalisierten im Wohnungsmarkt abzubauen resp. zu verhindern.

\section{Feministische Ansätze}

Im Rahmen feministischer sozialwissenschaftlicher Theorieansätze wird die besondere soziale Situation von Frauen im Rahmen von Situations-, Dekonstruktions- und Rekonstruktionsanalysen beschrieben und erklärt (vgl. Rodenstein 1993). Innerhalb dieses gemeinsamen Nenners unterscheiden 
sich die Zugänge jedoch erheblich, was sich vor allem in der unterschiedlichen Sicht auf die Ursachen und die Vorstellungen zum Ausmaß der Interventionen ausdrückt. ${ }^{22}$

Rodenstein (1994: 16-19) unterscheidet einen liberalen, einen soziaiistischen und einen radikalen Flügel des Feminismus, wobei im Rahmen des ersteren vor allem die Art der staatlichen Regulation (durch Gesetzgebungen, Planungen und Verordnungen) als ursächlich für die Benachteiligung der Frauen angesehen wird. Der sozialistische Ansatz ist kapitalismuskritisch und sieht die Benachteiligung der Frauen als Folge einer doppelten Unterdrückung vermittels des kapitalistisch organisierten (Erwerbs-)Arbeitsmarktes und des Patriarchats, welche die meist unbezahlte Reproduktionsarbeit einseitig den Frauen aufbürdet (vgl. Werlhoff et al. 1983, BeckerSchmidt 1988). In diesem Ansatz wird vor allem der Zusammenhang von 'gender' und 'class' betrachtet. Die Vertreterinnen des radikalen Flügels analysieren die sozialen Konstruktionen und Rekonstruktionen geschlechtlicher Unterschiede - oftmals über den sozialistischen Ansatz hinausgehend - in ihren komplexen Macht-, Herrschafts- und Ausbeutungsverhältnissen und wollen mit ihrer Forschung einen aktiven Beitrag zu deren Überwindung leisten. Diese Art feministischer Forschung ist also bezüglich der sozialen Ungleichheiten und deren Abbildung in den Raum nicht wertfrei sondern dezidiert parteiisch.

Die »doppelte Vergesellschaftung« der Frauen wird von Lenz (1995, 3338) zur »dreifachen Vergesellschaftung der Frau« erweitert. Sie fügt als dritte Dimension der unterdrückenden Vergesellschaftung die »Nationalstaaten der Moderne« an, die bezüglich der MigrantInnen die Dazugehörigkeit regeln und damit eine (weitere) Ebene der »Segregation « - gemeint ist: Segmentation - ausbilden. Dieses weitere Argument scheint mir kein für die Geschlechterverhältnisse spezifisches zu sein, wohl aber das Beispiel, das sie an anderer Stelle gibt. Der Zugang zu staatlich/kommunal vermittelten gesellschaftlichen Ressourcen. Darunter kann instrumentell und an Alltagspraktiken orientiert (öffentlich geförderter und zugewiesener) Wohnraum, Infrastruktureinrichtungen, Erreichbarkeit etc. verstanden werden, oder aber theoretisch: der Raumbezug. Das bedeutet, daß die »dritte Vergesellschaftungsform《 über die soziale Ungleichheit hinausgehend für die Segregation von besonderer Relevanz ist.

Die MehrzahI der stadtplanerischen und stadt- und regionalsoziologischen Ansätze feministischer Forschung dürften dem radikalen Flügel zuzu-

22 An dieser Stelle soll und kann keine differenzierte Darstellung femistischer Theorien vorgenommen werden (vgl. hierzu: Brück et al. 1992, Becker-Schmidt/Knapp 1995). Hier geht es ausschließlich darum, die Ansätze zur Beschreibung, Erklärung sowie die Bewertung der Folgen zum Phänomene der Segregation zusammenzustellen. 
rechnen sein; einige Vertreterinnen von ihnen entstammen ursprünglich der materialistischen Denktradition und argumentieren demzufolge auch klassentheoretisch und/oder aus der Sicht der Regulationstheorie. Gleichwohl »... stehen Erfolge in der feministischen Theorie des Planens und Bauens noch weitgehend aus« (Rodenstein 1994, 25).

\subsection{Beschreibung der Segregation}

Die Beschreibung sozialer Ungleichheiten innerhalb der feministischen Stadt- und Regionalsoziologie richtet sich vor allem auf die eingeschränkten Zugangs- und Aneignungsmöglichkeiten von Raum für Frauen (»dritte Vergesellschaftung «) und zwar bezüglich:

- der Verweigerung des Zugangs zum öffentlichen, und damit gesellschaftlichen und politischen Raum,

- der Verweisung auf periphere und damit auf schlechter erreichbare und monotoner ausgestattete Räume,

- der Verweisung auf kleinere (bei Sport, Spiel, Aktionsräumen etc.), aber auch für spezifische Nutzungen stärker determinierte Räume (z.B. von Männern ergonomisch gestaltete Küchen als Arbeitsraum für eine isolierte Person) sowie das Fehlen von Rückzugsräumen.

In diesem Zusammenhang wird als Segregationsaspekt vor allem die benachteiligte Rolle der Hausfrauen, der Teilzeit-Erwerbstätigen und der Alleinerziehenden in peripheren Wohnstandorten betrachtet. Durch eine eher schlechte Infrastrukturausstattung, in der Regel ohne Zugriff auf einen Pkw, und daher auf Fußmärsche, Fahrradfahrten oder den öffentlichen Nahverkehr angewiesen, bewirken die durch Planung und Marktgesetze in einer fordistisch organisierten Stadt der Nutzungstrennung errichteten Raum(räumliche Distanzen) und Zeitstrukturen (Öffnungszeiten und Wegedauer) für Frauen hohe ökologische Distanzen, die entweder nicht oder nur unter sehr hohen zeitlichen und finanziellen Opfern überwunden werden.

»Die fortschreitende räumliche Trennung von 'home' und 'work', des Wohnens und damit der unbezahlten Hausarbeit von der bezahlten Erwerbsarbeit, wird als 'geschlechtsspezifische' Segregation des Stadtraums angesehen, die zu einem wichtigen Merkmal der funktionalen Differenzierung des Stadtraums geworden ist. Geschlechtsspezifische Rollenzuweisungen ... spiegeln sich in der geschlechtsspezifischen Raumaufteilung der Stadt wider (Borst 1990, 246).

Borst (1990, 250-262) betont als einen zweiten Aspekt der 'gender segregation' die Ausdifferenzierung der Lebensverhältnisse von Frauen. Die Flexibilisierungen des Arbeitsmarktes, die zunehmende Stärkung des tertiären Sektors und nicht zuletzt das ausgeweitete Bildungssystem führt zu stärkeren Erwerbsbeteiligung von Frauen, allerdings vor dem Hintergrund sich ausweitender Disparitäten zwischen den Frauen (die »Zuverdienerinnen«, oftmals Migrantinnen, erledigen einen Teil der Reproduktionsarbeit der karriereorientierten Frauen). Das führt zu einer Polarisierung und Hetero- 
genisierung weiblicher Alltagserfahrungen und Raumnutzungsmuster durch die Segregation zwischen Familien an der Peripherie (mit der Hausfrau als Reproduktionsarbeiterin) und Nicht-Familien in innenstadtnahen Quartieren (mit in der Regel erwerbstätigen Frauen, die - höhere Einkommen vorausgesetzt - sich von der Reproduktionsarbeit teilweise freikaufen und die verbleibende in einem Partnerhaushalt eher gleichberechtigt aufteilen).

Alisch (1993, 114-125) nimmt diese Argumente auf, indem sie Gentrification als »räumliche Konsequenzen der veränderten Frauenrolle(n)« ansieht. Zusätzlich beschreibt sie neben der Segregation entlang unterschiedlich emanzipierter Frauenrollen im Sinne eines Zentrum-Peripherie-Modells die Konkurrenz zwischen Frauen in unterschiedlicher sozialer Lage. Hierzu unterscheidet sie vor allem aufgrund der unterschiedlichen Ausstattung mit ökonomischem und kulturellem Kapital in Zuverdienerinnen, Doppelverdiener- und Doppel-Karriere-Haushalte, wobei letztere - in der Regel als kinderlose Haushalte (DINKS oder 'empty nesters') - die größten Durchsetzungschancen am Wohnungsmarkt aufweisen. In den innerstädtischen Quartieren, deren Aufwertungsprozesse bereits eine Weile andauern, lassen sich auch Zeichen einer 'family gentrification' nachweisen, d.h. die Frauen bekommen in der Regel noch recht spät ein oder zwei Kinder, ohne daß sie deswegen an die Peripherie umziehen. Wie empirische Untersuchungen in mehreren Hamburger innenstadtnahen Wohnquartieren gezeigt haben, liegt der Anteil der Frauen, die sich selbst als Hausfrauen einstufen, trotz der family gentrification' unter 10\% (vgl. Dangschat/Alisch 1995).

\subsection{Ursachen der Segregation}

Die Ursachen der Segregation werden einerseits in den strukturellen Benachteiligungen der Frauen an sich gesehen, andererseits in den zunehmend differenzierten Lebenslagen zwischen den Frauen aufgrund der Veränderungen des Arbeitsmarktes und allgemeiner Modernisierungsprozesse, zu denen auch der Rückgang der Kleinfamilie sowie das In-Frage-Stellen traditioneller Verteilungen der Geschlechterrollen gehört. Dennoch geht in Deutschland wohl keine Autorin so weit wie die Amerikanerin Markusen $(1981,31)$, die in der Gentrification $» . .$. in weiten Teilen ein Resultat des Zusammenbruchs der patriarchalen Haushalte« erkennt.

Für die prinzipielle Benachteiligung von Frauen durch städtebauliche Mängel werden vor allem die Regulationsweise und die Konsummodelle des Fordismus herangezogen. Die hohe Bewertung individueller Mobilität durch die Verfügung über einen Pkw (»Autogerechte Stadt «), die Eigenheim- und patriarchale Kleinfamilien-Ideologie haben sich in einer hohen NutzungsSegmentierung und insbesondere in Suburbanisierungen niedergeschlagen. Diese »Produktion und Reproduktion patriarchaler Beziehungen durch die 
baulich-räumlichen Strukturen unserer Städte« hat vor dem Hintergrund der Zuweisung der unentgeltlichen Reproduktionsarbeit an Frauen »... das Alltagsleben von Frauen erschwert, geschlechtsspezifische Rollenzuweisungen befestigt und den Frauen Wahlmöglichkeiten zur Erweiterung ihrer Aktivitätsräume« beschnitten (Borst 1990, 237; vgl. auch Rodenstein et al. 1996).

Die zweite Art der 'gender segregation' thematisiert die räumliche Konzentration von Frauen in bestimmten Lebenslagen: die Konzentration armer Frauen (Alleinerziehende, Mütter großer Familien, allein lebende ältere Frauen) in den Großsiedlungen, die der Hausfrauen an der Peripherie (Suburbanisierung, zusätzlich überlagert durch erhebliche Einkommensunterschiede) und die der karriereorientierten Alleinlebenden oder in Partnerschaft lebenden (Gentrification). Dabei entspricht die Suburbanisierung der fordistischen Phase, während die Gentrifizierung alle Aspekte der Modernisierung unter einem postfordistischen Regime in sich vereint. Die Konzentration von Armut und sozialer Problematik ist wiederum Folge der Umstrukturierung im Zuge des Übergangs von der fordistischen zu einer nachfordistischen Produktions-, Reproduktions- und Regulationsweise des Arbeitsmarktes und des Raumes.

Sowohl die insbesondere über den Arbeitsmarkt sozial differenzierte und zunehmend polarisierte Lebenssituation von Frauen, als auch die Produktion von Raum wird von Borst (1990) als Folge eines langandauernden fordistischen Regimes und zuletzt des (krisenhaften) Übergangs im Rahmen der Flexibilisierung des Fordismus angesehen. In diesem Zusammenhang verstärkt sich die »'Durchkapitalisierung' der Reproduktionssphäre« (Borst 1990, 239; vgl. auch Rodenstein et al. 1996), was sich insbesondere in den Metropolen zeigt. Vor dem Hintergrund polarisierter Sozial- und Raumstrukturen sind die neuen Formen der Stadtpolitik und Stadtplanung Instrumente der Produktion und Reproduktion neuer sozialräumlicher Ungleichheiten zwischen den Geschlechtern, aber auch der Frauen untereinander (Borst 1993). Damit entspricht das Erklärungsmodell zugespitzter sozialräumlicher Ungleichheiten weitgehend dem des polit-ökonomischen Ansatzes resp. dem des Regulationsansatzes, wobei jene nach wie vor die $\mathrm{Ge}-$ schlechterverhältnisse allenfalls am Rande berücksichtigen.

\subsection{Folgen der Segregation und deren Bewertung}

Die Folgen der funktionalen Entmischung sowie der Gentrification und Segregation von weiblichen Lebenslagen werden von feministischen Autorinnen durchgängig als negativ angesehen, weil sie eine zusätzliche Benachteiligung der Frauen aufgrund deren Zuweisung zu benachteiligenden Wohnungs- und Wohnumfeldbedingungen beinhalten. D.h. die Einschätzung der Segregation resp. der Zuweisung von Orten bestimmter Qualitäten und La- 
gen reproduziert geschlechtsspezifische Ungleichheiten und führt zu steingewordenen, patriarchalisch geprägten Alltagskontexten, die eine zusätzliche Schwierigkeit darstellen, die Reproduktionsarbeit zu bewältigen bzw. mit der Zuweisung von Doppel- und Mehrfachbelastungen umgehen zu können.

\subsection{Interventionen in den Segregationsprozess}

Die Möglichkeiten in die Gestaltung der Orte zu intervenieren, die für Frauen (zusätzlich) benachteiligend sind, wird vor allem im Rahmen einer veränderten Stadtplanung und Architektur erwartet. Über eine stärkere Beteiligung von Frauen an der Architektur, der Modernisierung von Wohnraum und Stadtteilen sowie der Stadtplanung soll ein Freiraum geschaffen werden, um Bedürfnisse von Frauen an das Wohnen und das Wohnumfeld mit (anderen) Frauen zu diskutieren, zu formulieren und umzusetzen. Dieses sind in der Regel liberale Zugänge, d.h. unter Ausnutzung institutioneller Machtzuwächse soll eine Gegenplanung installiert werden, die nicht die grundsätzlichen geschlechtsspezifischen Ungleichheiten aufhebt, sondern die zusätzliche Diskriminierung der Reproduktionsarbeitsplätze und die Privatisierung der weiblichen Alltagspraktiken zu vermeiden sucht. Die radikalen Positionen im Bereich der feministischen Stadt- und Regionalsoziologie, von der eingangs die Rede war, konzentrieren sich eher auf die Ursachendiskussion, was wohl ein Grund für deren mangelnde Unterstützung durch die Planung ist, auf die Rodenstein (1994) hinweist.

\section{Zusammenfassung und Kritik}

In aller Knappheit wurde versucht, mit der residentiellen Segregation eine zentrale Kategorie der Stadt- und Regionalsoziologie aus der Sicht der gegenwärtigen »Schulen« darzustellen. Jede von ihnen hat eine spezifische Stärke: die der Sozialökologie liegt in der Beschreibung, Messsung und Kartierung, die des polit-ökonomischen Ansatzes liegt in der Makro-Erklärung, während die feministische Stadt- und Regionalsoziologie vor allem die Folgen (allerdings überwiegend nur für Frauen) reflektiert.

Nicht zuletzt deshalb ist die Einsicht gewachsen, daß mit keinem der drei Ansätze für sich genommen das Phänomen in ausreichender Weise empirisch beschrieben, erklärt, in seinen Folgen diskutiert und womöglich gar eine Prognose gewagt werden kann. Nachdem Friedrichs (1988b) das Ende der klassischen Sozialökologie eingeräumt hat (sie habe »ausgedient«), glaubt auch Hamm $(1994,216)$, daß man - um $» .$. Stadtentwicklung verstehen zu können« - »... (analytisch) auch nationale und globale Entwicklungen verstehen《 können müßte. Selbstkritisch fügt er hinzu: »Vielmehr haben wir uns ... einem positivistischen Wissenschaftsverständnis unter- 
worfen, daß sich angesichts der globalen Bedrohung als weltweit irrelevant erweist « (Hamm 1994, 220).

Auch die feministische Stadt- und Regionalsoziologie ist zu schmal konzipiert, wenn sie sich ausschließlich um die Übertragung der Geschlechterbeziehung in den Raum als einer Ursache zur Reproduktion sozialer Benachteiligung von Frauen beschränkt. Aufgrund der Ausbildung meist in sozialökologischer oder polit-ökonomischer Weise werden jedoch ohnehin zu sätzliche Mikro- resp. Makro-Aspekte zur Analyse der Segregation herangezogen.

Der polit-ökonomische Ansatz verfügt zwar - zunehmend mit Hilfe des Rückgriffs auf die Regulationsschule(n) - über einen umfangreichen Erklärungsapparat der ökonomischen und politischen Kontexte des sozialen Wandels, doch sind die »Anschlußtheorien« zur Meso- und Mikro-Ebene häufig unklar. Das drückt sich auch darin aus, daß das Phänomen der residentiellen Segregation nicht einmal hinreichend beschrieben wird und die Muster der sozialräumlichen Differenzierung eher ex-ante bestimmt, denn empirisch ermittelt werden.

Jedem Ansatz für sich, aber auch allen bislang vorliegenden Zusammenführungen fehlt eine überzeugende Darstellung der drei, für eine Segregationstheorie grundlegenden Module: eine Theorie sozialer Ungleichheit, eine Theorie räumlicher Ungleichheit und eine Theorie der Zuweisung von Wohnraum und Stadtteilen an Haushalte und soziale Aggregate. Erst mit der Konfiguration aus sozialen und räumlichen Strukturen sowie deren Interdependenzen kann erreicht werden, was Marcuse (1989) in seinem »quartered city«-Ansatz fordert (aber selbst nicht einlöst): Ein wissenschaftliches Verständnis für das Wechselverhältnis ökonomischer und politischer Prozesse, individueller Entscheidungen und der Bedeutung städtischer Teilräume und ihrer Akteure, die in ihren funktionalen Bezügen und regionalen Anordnungen einander wechselseitig bedingen.

Eine Theorie der residentiellen Segregation umfaßt längst nicht mehr nur noch eine Übertragung sozialer Ungleichheit in den Raum oder dient als Indikator für soziale Segmentation, sondern die Segregation (und in ihr Prozesse der Konzentration spezifischer sozialer Aggregate) ist ein entscheidender Motor für die Reproduktion sozialer Ungleichheit, indem sozial Benachteiligte in diejenigen Wohn- und Wohnumfeldsituationen abgedrängt werden, in denen sie keine gleichen Entwicklungschancen haben, weil sie von vielen städtischen Einrichtungen aufgrund schlechter Ausstattung und mangelnder Erreichbarkeit abgeschnitten und die Emissionen höher sind. Zudem sind soziale Netzwerke dort nur schwierig als »soziales Kapital« zu entwickeln, wo die Wertevielfalt groß, der kulturelle Hintergrund verschieden, die individuellen und familialen Probleme jedoch groß sind. 
Segregationsanalysen gleich welcher Herkunft sollten jedoch nicht nur das Ausmaß und die Art der Segregation sowie deren räumliche Ausprägungsmuster beschreiben und die Arbeits- und Wohnungs»markt«entwicklung sowie deren politische Regulation kritisieren, sondern auch den Umgang mit sozialräumlichen Konzentrationen sozialer Gruppen analysieren. Wenn es richtig ist, daß Konzentrationen intensiver ausfallen, als es dem Toleranzniveau und den Interessen der Bevölkerung entspricht, dann ist zu fragen, wie die Toleranz gestärkt und ausgeweitet werden kann. Dort, wo die Filterungsprozesse nach unten bereits sehr stark sind (weil zu viele »Fremde« einziehen, weil die Bodenpreise verfallen etc.), dort gilt es, die Flüchtenden von der Notwendigkeit zum Bleiben zu überzeugen.

Vor dem Hintergrund weitaus überwiegender Bestandspolitik (gegenüber dem Neubau), sind die angemessenen normativen Fragen nicht mehr nach der maximalen Zahl einer Sorte Menschen, die einem Hauseingang, einem Wohnblock oder einem Viertel »zugemutet« werden sollten, sondern die Frage, welche Art und auf welchem Niveau sozio-ökonomische und soziokulturelle Heterogenität erhalten werden sollte und kann. Denn auch der idealtypische Zugang Parks fragte eigentlich danach, was die Kräfte sind, die dazu führen, daß es 'natural areas' nicht gibt.

\section{Literatur}

Abu-Lughod, Janet (1969): Testing the Theory of Social Area Analysis: The Ecology of Cairo, Egypt, in: American Sociological Review, Vol. 34: 198-212.

Alisch, Monika (1993): Frauen und Gentrification. Der Einfluß von Frauen auf die Konkurrenz um den innerstädtischen Wohnraum. Leverkusen: Deutscher Universitätsverlag.

-; Dangschat, Jens S. (1993): Die solidarische Stadt. Ursachen von Armut und Strategien für einen sozialen Ausgleich. Darmstadt: Verlag für wiss. Publikationen.

-; Dangschat, Jens S. (1996): Die Akteure der Gentrifizierung und ihre »Karrieren«, in: Friedrichs, Jürgen; Kecskes, Robert (Hrsg.): Gentrification. Theorien und Forschungsergebnisse. Opladen: Leske + Budrich: 95-129

Amir, Y. (1969): Contact Hypothesis in Ethnic Relations, in: Psychological Bulletin, Vol. 71 , No. 5: 319-342.

Atteslander, Peter; Hamm, Bernd (Hrsg.) (1974a): Materialien zur Siedlungssoziologie. Köln: Luchterhand.

-; - (1974b): Einleitung: Grundzüge einer Siedlungssoziologie. in: dies. (Hrsg.) (1974a): 11-32.

Becker-Schmidt, Regina (1988): Perspektiven einer feministischen Theorie gesellschaftlicher Reproduktion, in: Sektion Frauenforschung (Hrsg.): Frauenforschung - Frauenpolitik. Hannover.

-; Knapp, Gudrun-Axeli (Hrsg.) (1995): Das Geschlechteverhältnis als Gegenstand der Sozialwissenschaften. Frankfurt am Main; New York: Campus.

Berger, Peter A.; Hradil, Stefan (1990): Die Modernisierung sozialer Ungleichheit und die neuen Konturen ihrer Erforschung, in: Berger, Peter A.; Hradil, Stefan (Hrsg.): Lebenslagen, Lebensläufe, Lebensstile. Soziale Welt, Sonderband 7. Göttingen: Otto Schwartz: 3-24.

Berger, Peter A.; Vester, Michael (Hg.) (1997): Alte Ungleichheiten. Neue Spannungen. Sozialstrukturanalyse 11, Opladen: Leske \& Budrich.

Blanke, Bernhard; Evers, Adalbert; Wollmann, Hellmut (Hrsg.) (1986): Die zweite Stadt. Neue Formen lokaler Arbeits- und Sozialpolitik, Leviathan, Sonderheft 7. Opladen: Westdeutscher Verlag.

Blasius, Jörg (1988): Indizes der Segregation, in: Friedrichs (Hrsg.) (1988): 410 431. 
- (1993): Gentrification und Lebensstile. Eine empirische Untersuchung. Wiesbaden: Deutscher Universitätsverlag.

-; Dangschat, Jens S. (Hrsg.) (1990): Gentrification - Die Aufwertung innenstadtnaher Wohngebiete. Frankfurt am Main: Campus.

-; Dangschat, Jens S. (1991): An Explanation of Residential Segregation by Education for One City. The Case of Warsaw, in: Journal of Urban Affairs, Vol. 13, No. 3: 353-366.

Borst, Renate (1990): Die zweite Hälfte der Stadt. Suburbanisierung, Gentrifizierung und frauenspezifische Lebenswelten, in: Borst et al. (Hrsg.) (1990): 235-268.

- (1993): Frauen und sozialräumliche Polarisierung der Stadt, in: FOPA Berlin (Hrsg.) 1993: $71-101$.

-; Krätke, Stefan; Mayer, Margit; Roth, Roland; Schmoll, F. (Hrsg.) (1990): Das neue Gesicht der Städte. Theoretische Ansätze und empirische Befunde aus der internationalen Debatte. Basel et al.: Birkhäuser.

Bourdieu, Pierse (1982): Die feinen Unterschiede. Kritik der gesellschaftlichen Urteilskraft. Frankfurt am Main: Suhrkamp.

- (1983): Ökonomisches Kapital, kulturelles Kapital und soziales Kapital, in: Kreckel, Reinhard (Hrsg.): Soziale Ungleichheiten. Soziale Welt, Sonderheft 2. Göttingen: OttoSchwarz-Verlag: 183-198.

- (1991): Physischer, sozialer und angeeigneter physischer Raum, in: Wentz (Hrsg.) (1991): 25-34.

Brück, Brigitteu.a. (1992): Feministische Soziologie. Eine Einführung. Frankfurt am Main; New York: Campus.

Coleman, James (1990): Foundations of Social Theory. Cambridge, M.A.: Belknap Press.

Dangschat, Jens S. (1985): Soziale und räumliche Ungleichheit in Warschau. Hamburg: Christians.

- (1988): Gentrification: der Wandel innenstadtnaher Wohnviertel, in: Friedrichs (Hrsg.) 1988: 272-292.

- (1990): Geld ist nicht (mehr) alles - Gentrification als räumliche Segregierung nach horizontalen Ungleichheiten, in: Blasius; Dangschat (Hrsg.) 1990: 69-92.

- (1991): Gentrification - Indikator und Folge globaler ökonomischer Umgestaltung, des sozialen Wandels, politischer Handlungen und von Verschiebungen auf dem Wohnungsmarkt in innenstadtnahen Wohngebieten. Unveröff. Habilitationsschrift. Hamburg: Universität Hamburg.

- (1994a): Lebensstile in der Stadt. Raumbezug und konkreter Ort von Lebensstilen und Lebensstilisierungen, in: Dangschat/Blasius (Hrsg.) (1994): 335-354.

- (1994b): Segregation - Lebensstile im Konflikt, soziale Ungleichheiten und räumliche Disparitäten, in: Dangschat/Blasius (Hrsg.) (1994): 426-445.

- (1995): "Stadt« als Ort und als Ursache von Armut und sozialer Ausgrenzung, in: Aus Politik und Zeitgeschichte, B 31-32/95: 50-62.

- (1996): Raum als Dimension sozialer Ungleichheit und Ort als Bühne der Lebensstilisierung? - Zum Raumbezug sozialer Ungleicheit und von Lebensstilen, in: Schwenck, Otto G. (Hrsg.): Lebensstil zwischen Sozialstrukturanalyse und Kulturwissenschaft. Opladen: Leske + Budrich: 99-135.

- (1997a): Klassenstrukturen im Nach-Fordismus, in: Berger/Vester (Hg.) (1997).

- (1997b): Warum ziehen sich Gegensätze nicht an? Zu einer Mikro-Makro-Theorie ethnischer und rassistischer Konflikte im Raum, in: Backes, Otto; Dollase, Reiner; Heitmeyer, Wilhelm (Hrsg): Ethnisch-kulturelle Konflikte in der Stadt. Frankfurt am Main: Suhrkamp. $\operatorname{lm}$ Druck.

-; Alisch, Monika (1995): Gentrification in Hamburg. Die ökonomische Aufwertung und kulturelle Umwertung dreier innenstadtnaher Wohngebiete. Unveröff. DFG-Abschlußbericht Da 219/1-2.

-; Blasius, Jörg (1987): Social and Spatial Disparities in Warsaw in 1978: An Application of Correspondence Analysis to a 'Socialist City', in: Urban Studies, Vol. 24: 173-191.

-; Blasius, Jörg (Hrsg.) (1994): Lebensstile in den Städten. Konzepte und Methoden. Opladen: Leske + Budrich.

-; Friedrichs, Jürgen (1988): Gentrification in der inneren Stadt von Hamburg. Hamburg: Gesellschaft für sozialwissenschaftliche Stadtforschung. 
-; Friedrichs, Jürgen; Kiehl, Klaus; Schubert, Klaus: (1985): Phasen der Landes- und Stadtentwicklung, in : Friedrichs, Jürgen (Hrsg.): Stadtentwicklungen in West-und Osteuropa. Berlin; New York: de Gruyter: 1-148.

Dear, Michael J.; Wolch, J.R. (1991): Wie das Territorium gesellschaftliche Zusammenhänge strukturiert, in: Wentz (Hrsg.) (1991): 233-247.

Dörrhöfer, Kerstin (Hrsg.) (1990): Stadt - Land - Frau. Soziologische Analysen - feministische Planungsansätze. Freiburg i. Br.: Kore.

Esser, Hartmut (1988): Sozialökologische Stadtforschung und Mehr-Ebenen-Analyse, in: Friedrichs (Hrsg.) (1988): 35-55.

Esser, Josef; Hirsch, Joachim (1987): Stadtsoziologie und Gesellschaftstheorie. Von der Fordismuskrise zur »postfordistischen« Regional- und Stadtstruktur, in: Prigge, Walter (Hrsg.): Die Materialität des Städtischen. Stadtentwicklung und Urbanität im gesellschaftlichen Umbruch. Stadtforschung aktuell, Bd. 17. Basel et al.: Birkhäuser: 31-58.

FoPa Berlin (Hrsg.) (1993): Frei - Räume. Raum greifen und Platz nehmen. Streitschrift der Feministischen Organisation von Planerinnen und Architektinnen - FOPA e.V., Sonderheft 1992/93, Zürich; Dortmund: eFeF-Verlag.

Friedrichs, Jürgen (1972): Großstadtforschung in den USA, in: Pfeil, Elisabeth (Hrsg.): Großstadtforschung. Hannover: Gebr. Jänecke Verlag: 100-110, 2. Aufl.

- (1977): Stadtanalyse. Soziale und räumliche Organisation der Gesellschaft. Opladen: Westdeutscher Verlag.

- (Hrsg.) (1988a): Soziologische Stadtforschung. Sonderheft 29/1988 der Kölner Zeitschrift für Soziologie und Sozialpsychologie. Opladen: Westdeutscher Verlag.

- (1988b): Stadtsoziologie - wohin? in: Friedrichs (Hrsg.) 1988a: 7-17.

- (1988c): Makro- und mikrosoziologische Theorien der Segregation, in: ders. (Hrsg.) (1988a): 56-77.

- (1995): Stadtsoziologie. Opladen: Leske + Budrich.

Gans, Herbert J. (1961): The Balanced Community; Homogeneity or Heterogeneity in Residential Areas?, in: Journal of the American Association of Planners, Vol. 27: 176-184.

Häußermann, Hartmut (1991): Sozialraum Stadt: Von der Sprengkraft sozialer Schichtung, in: Ganser, Karl; Hesse, Joachim Jens; Zöpel, Christoph (Hrsg.): Die Zukunft der Städte. Baden-Baden: Nomos: 92-110.

-; Siebel, Walter (1987): Neue Urbanität. Frankfurt am Main: Suhrkamp.

-; Siebel, Walter (1991a): Bausteine zu einem Szenario der Entwicklung von Berlin, in: Senatsverwaltung für Stadtentwicklung und Umweltschutz (Hrsg.): Metropole Berlin: Mehr als Marktl. Berlin: StadtUm: 23-58.

-; Siebel, Walter (1991b): Polarisierung der Städte und Politisierung der Kultur. Einige Vermutungen zur Zukunft der Stadtpolitik, in: Heinelt, Hubert; Wollmann, Hellmut (Hrsg.): Brennpunkt Stadt. Stadtpolitik und lokale Politikforschung in den 80er und 90er Jahren. Stadtforschung aktuell, Bd. 31, Basel et al.: Birkhäuser: 353-370.

Hamm, Bend (1977): Die Organisation der städtischen Umwelt. Ein Beitrag zur sozialökologischen Theorie der Stadt. Frauenfeld; Stuttgart: Huber.

- (1982): Einführung in die Siedlungssoziologie. München: Beck.

- (1994): Neue Aufgaben für sozialwissenschaftliche (Stadt-)Forschung, in: Meyer, Sybille; Schulze, Eva (Hrsg.): Ein Puzzle, das nie aufgeht. Stadt, Region und Individuum in der Moderne. Berlin: Edition Sigma: 209-226.

-; Neumann, Ingo (1996): Siedlungs-, Umwelt und Planungssoziologie. Opladen: Leske + Budrich; utb.

Herlyn, Ulfert (Hrsg.) (1974a): Stadt- und Sozialstruktur. München: Nymphenburger.

- (1974b): Einleitung: Wohnquartier und soziale Schicht, in: ders. (Hrsg.) (1974a): 16-41.

- (1974c): Soziale Segregation, in: W. Pehnt (Hrsg.): Die Stadt. Stuttgart: Reclam: 89-106.

- (1990): Stadtsoziologie in der Krise? Bemerkungen zu den Behauptungen von Jürgen Friedrichs, in: Kölner Zeitschrift für Soziologie und Sozialpsychologie, Jg. 42: 554-558.

Hitzler, Ronald (1994): Radikalisierte Praktiken der Distinktion. Zur Politisierung des Lebens in der Stadt, in: Dangschat/Blasius (Hrsg.) (1994): 47-58.

Hoffmeyer-Zlotnik, Jürgen (1977): Der Prozeß der Sukzession. Die Unterwanderung von Berlin-Kreuzberg. Hamburg: Christians. 
Hradil, Stefan (1987): Sozialstrukturanalyse in einer fortgeschrittenen Gesellschaft. Von Klassen und Schichten zu Lagen und Milieus. Opladen: Leske + Budrich.

- (1992): Alte Begriffe und neue Strukturen. Die Milieu-, Subkultur- und Lebensstilforschung der 80er Jahre, in: Hradil, Stefan (Hrsg.): Zwischen Bewußtsein und Sein. Opladen: Leske + Budrich: 15-55.

Kecskes, Robert; Knäble, Stefan (1988): Der Bevölkerungsaustausch in ethnisch gemischten Wohngebieten. Ein Test der Tipping-Theorie von Schelling, in: Friedrichs (Hrsg.) (1988a): 293-309.

Krätke, Stefan (1991): Strukturwandel der Städte. Städtesystem und Grundstücksmarkt in der "post-fordistischen Ara. Frankfurt am Main; New York. Campus.

- (1995): Stadt - Raum - Ökonomie. Einführung in aktuelle Problemfelder der Stadtökonomie und Wirtschaftsgeographie. Stadtforschung aktuell, Bd. 53. Basel et al.: Birkhäuser.

Läpple, Dieter (1991): Essay über den Raum. Für ein gesellschaftswissenschaftliches Raumkonzept, in: Häußermann, Hartmut u.a. (Hrsg.): Stadt und Raum - Soziologische Analysen. Pfaffenweiler: Centaurus: 157-207.

Lenz, Ilse (1995): Geschlecht, Herrschaft und internationale Ungleichheit, in: BeckerSchmidt; Knapp (Hrsg.) (1995): 19-46.

Marcuse, Peter (1989): 'Dual city': A Muddy Metaphor for a Quartered City, in: International Journal of Urban and Regional Research, Vol. 13: 697-708.

Markusen, Ann Roell (1981): City Spatial Structure, Women's Household Works, and National Urban Policy, in: Signs 5, Nr. 3: Supplement.

Marshall, Harvey; Jiobu, Robert (1975): Residential Segregation in the United States Cities. A Causal Analysis, in: Social Forces, Vol. 53: 449-460.

Müller, Hans-Peter (1992): Sozialstruktur und Lebensstile. Der neuere theoretische Diskurs über soziale Ungleichheit. Frankfurt am Main: Suhrkamp.

Park, Robert E. (1924): The Concept of Social Distance, in: Journal of Applied Sociology, Vol. 8.

- (1925): The Urban Community as a Spatial Pattern and a Moral Order, in: Publications of the American Sociological Association, Vol. 20: 1-14.

Rodenstein, Marianne (1993) : Feministische Stadt- und Regionalforschung - Zum Stand der Diskussion städtischer Lebensverhältnisse, in: FoPa Berlin (Hrsg.) (1993): 20-35.

- (1994): Wege zu einer nicht-sexistischen Stadt. Architektinnen und Planerinnen in den USA. Baden-Baden: Kore.

-; Bock, Stephanie; Heeg, Susanne (1996): In: Akademie für Raumforschung und Landesplanung (Hrsg.): Agglomerationsräume in Deutschland. Ansichten, Einsichten, Aussichten. Forschungs- und Sitzungsberichte, Band 199. Hannover: ARL: 26-50.

Roof, Wade C.; van Valey, Thomas L.; Spain, Daphne (1970): Residential Segregation in Southern Cities, in: Social Forces, Vol. 55: 59-71.

Saunders, Peter (1987): Soziologie der Stadt. Frankfurt am Main; New York: Campus.

Schelling, , Thomas C. (1971): Dynamic Models of Segregation, in: Journal of Mathematical Sociology, Vol. 1: 143-186.

Shevky, Eshref; Bell, Wendell (1955): Social Area Analysis. Stanford, Cal.: Stanford University. In deutscher Übersetzung in: Atteslander; Hamm (Hrsg.) 1974: 125-139..

Wentz, Martin (Hrsg.) (1991): Stadt-Räume: Frankfurt am Main; New York: Campus.

Werlhof, Claudia von; Bennholdt-Thomsen, Veronika; Mies, Maria (1983): Frauen - die letzte Kolonie. Zur Hausfrauisierung der Arbeit. Reinbek: Rowohlt. 